\title{
MAL DE POTT EN NIÑOS
}

\author{
PATRICIA CAMPOS* - EDUARDO CHAPARRO** - FEDERICO VALENCA**:; \\ ALFREDO FUENTES-DAVILA ***
}

\begin{abstract}
RESUMEN - Se presentan 27 casos de mal de Pott en niños entre 6 meses y 14 años de edad vistos durante un periodo de 2 años con tiempo de seguimiento hasta de 18 meses, $67 \%$ menores de 6 años $y$ de ellos 2 pacientes menores de 1 año. Hubo predominio de pacientes de sexo masculino. El sintoma “jiba” fue el mas frecuente. La localización mas frecuente de la lesión fue el nivel dorsal. Todos recibieron tratamiento médico con 4 drogas, 14 de ellos necesitaron de métudos ortopédicos $y$ solo 6 fueron sometidos a corrección quirúrgica. En mas del 50\% hubo una evolución favorable hacia la curación o la mejoria con secuelas.
\end{abstract}

\section{Pott's disease in children}

SUMMARY - Twenty seven cases of Pott's disease in children between 6 months and 14 years of age are reported. They were observed during a two years period, with a follow-up time till 18 months. In report to their ages $67 \%$ were lower than 6 years old and two of them lower than one year old. There was a male sex predominance. The 'hump' sign was the most frequently observed. The most frequent damage localization was at dorsal level. All patients received treatment with four drugs, 14 of them needed orthopedic methods, and only 6 wert: submitted to surgical correction. In more than $50 \%$ of the patients there was good outcome toward cure or improvement with sequelae.

Aunque la forma mas frecuente de tuberculosis (TBC) es la pulmonar, la mas severa es la de compromiso del sistema nervioso central (SNC). En 1951 ya Auerbach 1 citaba $42.2 \%$ de meningitis tuberculosa en una serie de 97 casos mortales de TBC en niños; y a partir de entonces diferentes autores se han ocupado de esta forma de compromiso cada vez mas frecuente. Sin embargo la forma de espondilitis o mal de Pott con o sin compromiso de paraplejia era descrita como complicación poco frecuente en la población pediátrica 3 . En décadas anteriores la mayor frecuencia era reportada en pacientes mayores de 16 años: Hodgson 10 en 1964, en una serie de 300 casos encuentra $43 \%$ menores de 5 años. Reportada como muy rara en lactantes que aun no caminan, la frecuencia en el grupo pediátrico viene aparentemente incrementándose en los últimos años, paralelo al incremento del compromiso del sistema nervioso en general 11,13,15,16. Usualmente resulta de diseminación hematógena en el curso de una infección tuberculosa inicial, algunas veces debida a drenaje linfático de otras áreas comprometidas como la pleura, o el rinón. En paises en desarrollo y en vias de desarrollo es responsable por aproximadamente $50 \%$ de la TBC osteoarticular, iniciándose entre $\operatorname{los} 2$ y 5 años.

Teniendo en cuenta la enorme trascendencia de la TBC en nuestros días y en países como el nuestro, y considerando que una lesión vertebral puede llegar a ser altamente invalidante, en el presente trabajo se revisan las caracteristicas clínicas del mal de Pott, se evaluan los resultados del tratamiento médico y ortopédico y/o quirúrgico en un seguimiento a mediano plazo y se intenta llamar la atención para la frecuencia creciente de esta afección en edades cada vez menores de la vida.

Hospital General Base Cayetano Heredia, Universidad Peruana Cayetano Heredia: * Neuropediatra, Departamento de Neurología; ** Pediatra, Departamento de Pediatría:

*** Neurocirujáno, Departamento de Cirugia. 


\section{MATERIAL E METODOS}

Se realiza un estudio retrospectivo y prospectivo de 27 pacientes entre 6 meses y 14 años de edad al momento de la primeira consulta, seleccionados por presentar una radiografia con lesión vertebral típica y uno o mas de los criterios que configuran su perfil tuberculoso: radiografía de tórax anomal PPD mayor de $10 \mathrm{~mm}$, mejoría radiológica luego de tratamiento, velocidad de sedimentación mayor de $12 \mathrm{~mm} / \mathrm{h}$, aislamiento (cultivo) de Mycobacterium tuber. culosis (BK) $y$ antecedentes epidemiologicos positivos de contacto tuberculoso o TBC extrapulmonar. Estos criterios se han modificado en base a los planteados por Konstam y Blesovsky 12

Dieciséis pacientes fueron vistos entre Septiembre de 1984 y Septiembre de 1986, las otros 11 fueran vistos en diferentes épocas. Solamente en 18 pacientes se pudo realizar seguimiento que varío entre 6 y 18 meses (muchos de ellos tenían seguimiento institucional hastia de 10 años). Los pacientes proceden de los Consultorios de Neurología y Pediatría del Hogar Clínica San Juan de Dios y del Consultorio de Neuropediatria del Hospital Cayetano Heredia.

Todos los pacientes fueron sometidos a examen clínico general, examen neurológico y examen psicométrico. Fueron realizados los seguientes exámenes auxiliares: radiografía de tórax, radiografía de columna A-P y lateral, PPD, velocidad de sedimentación; en 10 de ellos se realizó búsqueda de $B K$ en esputo $y$ en 4 se realizó punción percutánea de absceso osifluente. Se realizaron 10 mielografias con fimes quimúrgicos.

\section{RESULTADOS}

En relación a distribución por edad y sexo, encontramos 14 (52\%) de sexo masculino y $13(48 \%)$ de sexo feminino, distribuidos en 5 (19\%) lactantes (28 días a 2 años), 13 (48\%) pre-escolares (hasta 6 años) y 9 (33\%) mayores de 6 años. De los lactantes, 2 eran menores de 1 año, 6 y 10 meses respectivamente. El tiempo previo al diagnóstico vario entre 15 dias y 7 años, siendo en media 7.7 meses, encontrándose 20 (74\%) en el tiempo comprendido hasta el año, 4 pacientes tuvieron un tiempo mayor de 1 año y en 3 se desconoce el tiempo previo al diagnóstico. El sintoma mas frecuente de consulta fue la presencia de la clásica 'jiba' en 20 (74\%) pacientes, descrita como deformación progresiva de la columna en tiempos variados. Doce pacientes $(44 \%)$ presentaron dificultad progresiva para la marcha, 7 alza de temperatura no cuantificada o fiebre, en 3 hubo dolor. Outros sintomas referidos fueron: irritabilidad, convulsiones, déficit motor en un hemicuerpo y dificultad para lateralizar el cuello (Tabla 1). En relación ia los hallazgos del examen neurológico éste fue normal en 12 (44\%) pacientes, en $11(40 \%)$ hubo paraparesia o paraplejia, una paciente con lesión cervical presentó cuađriplegia; otros hallazgos fueron; signos meningeos en un paciente e hiperreflexia gIobłal en 2 pacientes.

Veinte pacientes tuvieron entre 1 y 4 criterios mas, al márgen de la radłografia vertebral típica, solo uno llenó los requisitos de tener los 6 criterios asociados. En orden de irecuencia, $16(59 \%)$ tuvieron radiografía de tórax anormal, 2 de ellos con patrón miliar, los dos menores de 5 años; $13(48 \%)$ mejoría radiológica luego de tratamiento, 14 (52\%) PPD mayor de $10 \mathrm{~mm}, 12(44 \%)$ VSG elevada, mayor de $12 \mathrm{~mm} / \mathrm{h}, 11(40 \%)$ antecedente de contacto TBC, dos de ellos además con TBC extra pulmonar en el momento (uno con osteomielitis y el otro con TBC ganglionar), en 4 se logró aislar el BK de material de absceso osifluente (Tabla 1). Es interesante señialar que 6 teniam historia negativa de contacto TBC y en 4 hubo antecedente de caída, previa al desarrollo de los sintomas (uno de ellos fue el de la lesión cervical con cuadriplejia). La localización del compromiso vertebral iue como sigue: 17 (63\%) dorsales con 10 de ellos dorsal baja, $8(30 \%)$ lumbares con igual número de lumbares altas y bajas $y$ 2 (7\%) cervicales. Ambos casos cervicales tuvieron localización en el mismo segmento (C4-C5). Hubieron 4 pacientes con compromiso de nas de un segmento vertebral y 10 con mas de 2 vértebras comprometidas.

Todos recibieron tratamiento médico en diferentes esquemas que incluyeron streptomicina, isoniacida, rifampicina, ethambutol y/o pirazinamida; 14 de ellos tratamiento ortopédico con corset de yeso o collarín y 6 fueron sometidos a corrección quirúrgica. Un seguimiento adecuado solo fue posible en 18 pacientes, encontrándose que: ninguno falleció, 3 (11\%) empeoraron luego de haber iniciado su recuperación (uno desarrolió TBC de rodilla, otro desarrollo paraplejia desde un examen previamente normal y el último tiene actualmente TBC multisistêmca); $7(29 \%)$ tuvieron evolución favorable incluyendo la paciente con paraplejia y uno con paraplejia que recuperaron ad integrum; 8 (30\%) tuvieron mejoria, quedando con secuelas leves a moderadas. (Tabla 1 ). 


\section{COMENTARIOS}

La osteomielitis vertebral secundaria a diseminación del bacilo tuberculoso en el niño era considerada complicación infrecuente en la literatura 2,9. Henderson (cit. por Bailey et al.2) encontró mayor incidencia en pacientes mayores de 25 años, Cleveland 5 , en pacientes entre 21-31 años. Manreza en 13 pacientes solo encuentra un paciente de 6 años 14. Si nos referimos únicamente a series pediátricas, Hodgson 10 en una serie de 300 casos tiene el $70 \%$ en menores de 10 años, Bailey et al. 2 encuentran que $76 \%$ de 100 pacientes consecutivos eran menores de 5 años. Nuestra casuística recoje 27 pacientes en un lapso de 2 años y en relación con la literatura $67 \%$ de ellos menores de 6 años. Un dato interesante es el de la escasa frecuencia de la afección en lactantes menores. Para Bailey et al.2 el menor de su serie tenía 1 año, y Dickson 6 solo tiene 2 lactantes de 31 pacientes pediátricos. Al igual que este último autor presentamos 2 lactantes menores ( 6 y 10 meses respectivamente).

Sorrel y Déjerine-Sorrel 18 clasificaron la paraplejia de acuerdo al intervalo entre el inicio de los síntomas y el inicio de la paraplejia, encontrando asi que no es posible establecer un paralelo. La espondilitis TBC compromete cualquier porción de la columna vertebral, siendo las mas frecuentes la región torácica baja seguida de la región lumbar $2,4,6,10$. Asi para Dobson 7 de 138 pacientes $57 \%$ eran de localización tóraco-lumbar, para Kaplan (cit. por Bailey et al.2) de 58 pacientes $60 \%$ eran torácicas. Bakalim (cit. por Bailey et al. ${ }^{2}$ ) asegura que en niños la localización torácica es dos veces mas frecuente que la lumbar. Nuestra casuistica sigue exactamente la misma distribución. Otro dato a ser considerado es el hecho de que múltiples compromisos vertebrales son mas frecuentes que el compromiso de una vértebra 18 , dato encontrado para 22 de nuestros pacientes.

De acuerdo con Seddon 17, la compresión medular ocurre en 10-25\% de los casos siendo la localización torácica la que mas se acompaña de compresión, sin embargo y a pesar de los pocos casos cervicales descritos, éstos generalmente se acompañan de mayor compromiso neurológico. Greenfield 9 consideraba que entre 5-20\% desarrollaba paraplejia en tanto que para Bailey et al. 2 este porcentaje sube a $43 \%$; ya para el estudio colaborativo realizado en Corea en 19768 se encontró solo $9 \%$ de paraparesia paraplejia. Uno de los dos casos cervicales fue el de mayor compromiso neurológico al presentar paraparesia crural severa y paraplejia braquial en nuestro estudio. Las manifestaciones clínicas en el niño dependen del estadio della enfermedad. Para Seddon 17 el dolor espontáneo o a la palpación es un rasgo prominente y Alvick (cit. por Manreza 14) lo encuentra en $70 \%$ de sus pacientes (no todos pediátricos); en casos avanzados se tiene la formación de la clásica jiba. Para nuestro grupo el dolor óseo no fue una constante, en tanto que la deformidad ósea estuvo presente en mas del $70 \%$, cifra comparable a la de Bailey et al. 2 con 38 de 43 pacientes con jiba. El mal de Pott se asocia frecuentemente con evidencia de TBC activa en pulmones; compromiso meníngeo no es sin embargo frecuente de ver; otros sistemas comprometidos tienen al génitourinario como el de mayor frecuencia. Bosworth y Levine 4 describen $10 \%$ de compromiso pulmonar. En la serie de Bailey et al. ${ }^{2}$ de 31 pacientes se describen 27 con lesión pulmonar. Sobre compromiso extra pulmonar, Bailey et al. 2 tienen 1 paciente con compromiso renal, Bosworth y Levine $10 \%$ de compromiso génitourinario y $6 / 177$ con compromiso de articulación de cadera 4. Para nuestra serie el compromiso pulmonar fue del orden de $59 \%, 1$ paciente desarrolló TBC multisistémica y otro TBC articular. Un paciente tuvo simultáneamente meningoencefalitis TBC y mal de Pott pero a pesar de ser descrita como asociación siempre fatal, el paciente mejoró.

Bosworth y Levine 4 en su serie de 122 pacientes y Bailey et al. 2 son unánimes en aceptar que los niños tienen un porcentaje mayor de recuperación favorable, un pronóstico mejor y una mejor capacidad de trabajo luego de la recuperación, cuando se comparan con series de pacientes de varias edades, independientemente de los tipos de tratamiento. Asi para nuestra casuistica, en el seguimiento prácticamente $88 \%$ tuvieron buena evolución, con $38 \%$ con evolución francamente favorable dentro de las que se cuentam las dos recuperaciones ad integrum. Finalmente y como en toda enfermedad infecciosa el diagnóstico precoz y el tratamiento adecuado y oportuno disminuye la morbimortalidad, y en ese sentido no podemos dejar de mencionar el hecho de que casi $75 \%$ acudieron a consulta con hasta 1 año de evolución del proceso, no obstante lo cual tuvimos un buen porcentaje de recuperación. 


\section{REFERENC1AS}

1. Auerbach $\mathrm{O}-$ Meningitis tuberculosa. Clin Med $\mathrm{N}$ Am 2:339, 1985.

2. Bailey HL, Sister MG, Hodgson AR, Shin JS - Tuberculosis of the spine in chitdren. J Bone Joint Surg 54A:1633, 1972.

3. Bell W, McCormick W - Neurologic Infections in Children. Ed 2. Sauders, Philadelphia, 1981, pag. 198.

4. Bosworth DM, Levine $\mathbf{J}$ - Tuberculosis of the spine: an analysis of cases treated surgically. J Bone Joint Surg 31A:267, 1919.

5. Cleveiand $\mathrm{M}$ - Tuberculosis of the spine: a clinical study of 203 patients from st Luke's Hospital. Am Rev Tuberc 11:215, 1940.

6. Dickon J A S - Spinal tuberculosis in Nigerian children: a review of ambulant treatment. $J$ Bone Joint Surg 49B:682, 1967.

7. Dobson $\mathrm{J}$ - Tuberculosis of the spine: an analysis of the results of consecutive treatment and of the factors influencing the prognosis. $J$ Bone Joint Surg 33B:517, 1951.

8. Fith Report of the Medical Research Councial Working - Party on Tuberculosis of the Spine - A five years assesnient of controlled trails of in-patient and out-patient treatment and of plaster-of-Paris jackets for tuberculosis of the spine in children on standard chemotherapy: studies in Masan and Pausan, Korea. J Bone Joint Surg 58B:399, 1976.

9. Grenfield $J$ G - Neuropathology. Edward Arnold, London, 1958.

10. Hodgson AR - Report of the findings and results in 300 cases of Pott's disease treated by anterior fusion of the spine. $J$ Western Pacific Orthop Ass 1:3, 1964.

11. Idriss $\mathrm{Z}$, Sinno A, Kronfol $\mathrm{N}$ - Tuberculous meningitis in children. Am J Dis Child $130: 364,1976$.

12. Konstam PG, Blesovsky A - The ambulant treament of spinal tuberculosis. Br J Surg $\check{0} 0: 26,1962$.

13. Lefèvre AB, Diament A. (eds) - Neurologia Infantil. Sarvier, São Paulo, 1980, pg 588.

14. Manreza LA - Tratamento da tuberculose espinal. Arq Bras Neurocir 3:141, 1985.

15. Martin NS - Tuberculosis of the spine. J Bone Joint Surg 52B:613, 1970.

16. Polo H, Cerna A, Diaz A, Apac A - Mal de Pott en niños. Acta Med Per 13:36, 1986.

17. Seddon HJ - Pott's paraplegia: prognosis and treatmente. Br J Surg 22:769, 1935.

18. Sorrel EM, Sorrel-Déjerine $Y$ - Recherches sur le transit du lipiodol par voie sous-arachnoidienne dans les differentes formes de paraplegie pottique. Rev Neurol (Paris) $2: 88,1924$. 